Thermal imaging investigation of modified fused silica at surface damage sites for understanding the underlying mechanisms of damage growth

R. A. Negres, M. W. Burke, P. DeMange, S. B. Sutton, M. D. Feit, S. G. Demos

November 3, 2006

Boulder Damage Symposium

Boulder, CO, United States

September 25, 2006 through September 27, 2006 
This document was prepared as an account of work sponsored by an agency of the United States Government. Neither the United States Government nor the University of California nor any of their employees, makes any warranty, express or implied, or assumes any legal liability or responsibility for the accuracy, completeness, or usefulness of any information, apparatus, product, or process disclosed, or represents that its use would not infringe privately owned rights. Reference herein to any specific commercial product, process, or service by trade name, trademark, manufacturer, or otherwise, does not necessarily constitute or imply its endorsement, recommendation, or favoring by the United States Government or the University of California. The views and opinions of authors expressed herein do not necessarily state or reflect those of the United States Government or the University of California, and shall not be used for advertising or product endorsement purposes. 


\title{
Thermal imaging investigation of modified fused silica at surface damage sites for understanding the underlying mechanisms of damage growth
}

\author{
R. A. Negres, M. W. Burke, P. DeMange, S. B. Sutton, M. D. Feit, and S. G. Demos \\ Lawrence Livermore National Laboratory \\ 7000 East Avenue, Livermore, CA 94551, USA
}

\begin{abstract}
We use an infrared thermal imaging system in combination with a fluorescence microscope to map the dynamics of the local surface temperature and fluorescence intensity under cw, UV excitation of laser-modified fused silica within a damage site. Based on a thermal diffusion model, we estimate the energy deposited via linear absorption mechanisms and derive the linear absorption coefficient of the modified material. The results indicate that the damage growth mechanism is not entirely based on linear absorption. Specifically, the absorption cross-section derived above would prove insufficient to cause a significant increase in the temperature of the modified material under nanosecond, pulsed excitation (via linear absorption at ICF laser fluences). In addition, irreversible changes in the absorption cross-section following extended cw, UV laser exposure were observed.
\end{abstract}

Keywords: fused silica, thermal and fluorescence imaging, surface irradiation effects of UV radiation, optical absorption coefficients

\section{INTRODUCTION}

It has been shown that laser-induced damage leads to the formation of a modified material containing various defect species as a result of exposure to extreme localized temperatures and pressures occurring during a damage event. In the case of fused silica, absorption of the laser light within this modified material has been suspected to play a key role in the damage growth process. A possible mechanism involves linear absorption leading to an increase in the temperature of the material above a characteristic temperature. Alternatively, one may need to consider nonlinear absorption mechanisms.

The objective of this work is to estimate the energy deposited under 351-nm CW excitation of laser modified $\mathrm{SiO} 2$ via linear absorption mechanisms. Our approach involves the use of an infrared thermal imaging system to map, with adequate spatial and temporal resolution, the dynamics of the local surface temperature of the sample. The laser beam was focused to $\sim 100 \mu \mathrm{m}$ within a single laser-induced damage (LID) site. Following the onset of UV laser irradiation and diffusion of energy deposited within the modified material, a temperature gradient was observed. The increase in surface temperature of the sample is a result of energy dissipation through non-radiative relaxation pathways by the absorbing defects in the modified material. A fluorescence microscope is also used to image the same sites in order to concurrently monitor the radiative relaxation pathways. Based on a thermal diffusion model and the kinetics of the measured surface temperature gradient, we calculate the fraction of energy deposited at damage sites following CW laser excitation and estimate the linear absorption coefficient of the modified material.

\section{EXPERIMENT}

We prepared LID sites at the exit surface of a fused silica substrate in air and at room temperature using 7-ns (FWHM), 355-nm laser pulses from a Q-switched Nd:YAG laser system (Spectra Physics, Model Quanta-Ray). These were typical surface damage sites initiated with one pulse at $20-30 \mathrm{~J} / \mathrm{cm}^{2}$ and subsequently grown to $\sim 500-800 \mu \mathrm{m}$ in diameter using ten pulses at $\sim 10 \mathrm{~J} / \mathrm{cm}^{2}$. A cw, Argon laser (351-363 nm) beam was centered and focused within a single site (HW1/eM of $110 \mu \mathrm{m})$. A mid-IR $(3-5 \mu \mathrm{m})$ imaging camera (Model Phoenix 9803,

Send correspondence to R. A. Negres: E-mail: negres2@llnl.gov 


\section{CW, multi-line Ar laser}

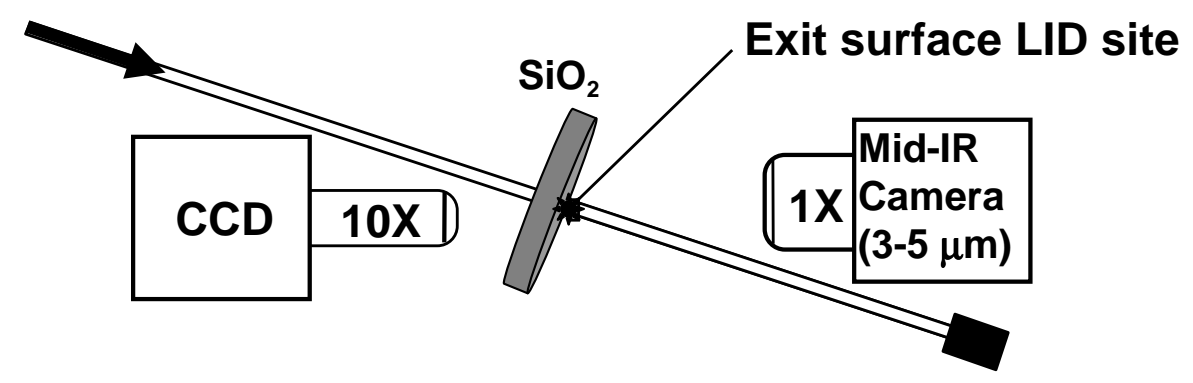

\section{Fluorescence imaging}

Thermal imaging

Figure 1. Schematic of thermal and fluorescence imaging setup for investigating energy deposition within laser-modified fused silica at surface damage sites.

Indigo Systems, with $640 \times 512$ cooled InSb sensor, $25 \mu \mathrm{m} /$ pixel, 14-bit dynamic range, full frame acquisition rates of up to 107 frames per second) equipped with $1 \times$ magnification optics was positioned behind the sample as illustrated in Fig. 1. We are thus able to directly image its back surface and spatially resolve changes in the surface temperature prior to, during and after laser irradiation. The temperature calibration of the IR camera was performed for each acquisition configuration using two methods: 1) a black body radiation source to derive the relative emissivity curve of fused silica, and 2) a thermo-couple device to directly measure the temperature of the IR-imaged sample from 20 to $60{ }^{\circ} \mathrm{C}$. The two calibration methods were in good agreement over this temperature range. In addition to monitoring the temperature, we also captured the fluorescence signal originating at LID sites during UV excitation using a fluorescence microscope placed in front of the sample, opposite to the IR camera, as shown in Fig. 1. A thermal map (with the laser OFF) and fluorescence image of the same LID site are illustrated in Fig. 2(a) and 2(b), respectively. The damage site is visible in Fig. 2(a) even without laser illumination due to perhaps its larger surface area compared to that of a pristine fused silica surface.
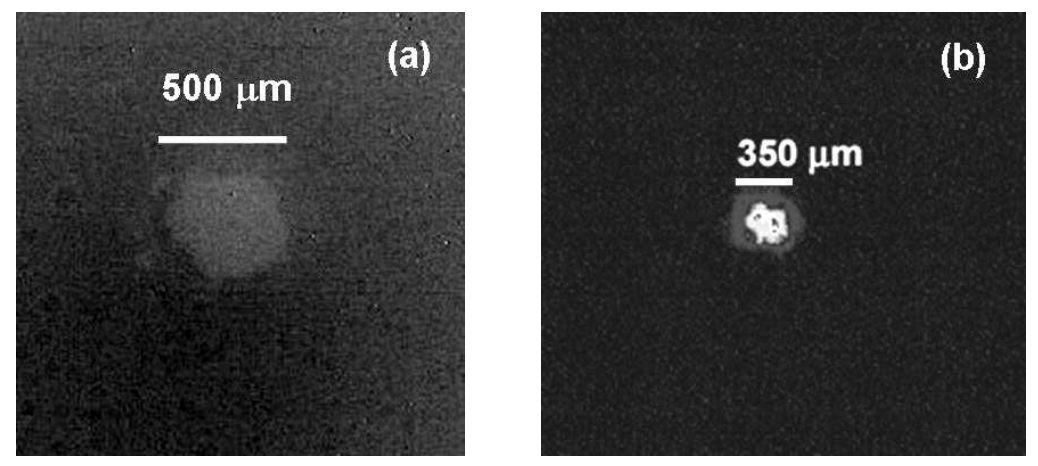

Figure 2. Thermal map (with laser OFF) and fluorescence image of a typical surface damage site.

Real time thermal imaging indicates that, following the onset of laser irradiation, a temperature gradient is rapidly established throughout the substrate due to diffusion of energy deposited within the modified material. Experiments were performed in two different irradiation configurations, either short or extended exposure to UV laser beam with constant power. 


\section{RESULTS AND DISCUSSION}

The first set of experiments was designed to resolve the temperature rise and fall at a damage site upon turning the laser ON and OFF using a mechanical shutter placed in the beam path. In this case, the sample was irradiated for few seconds and maximum spatial resolution thermal maps were acquired at 100 frames/sec with $0.5 \mathrm{msec}$ integration time for 12 seconds, including the brief exposure to laser radiation. We can then extract the local surface temperature at various locations relative to the LID site from individual frames and plot it versus time (corresponding to the frame number). Figure 3 illustrates the surface temperature change at three locations (center, $0.5 \mathrm{~mm}$ and $1.75 \mathrm{~mm}$ away, represented by circle, triangle and square data points, respectively) prior to, during and after constant laser irradiation at $2.6 \mathrm{~W}$. The temperature values represent the average values integrated over 4 pixels at the above locations.

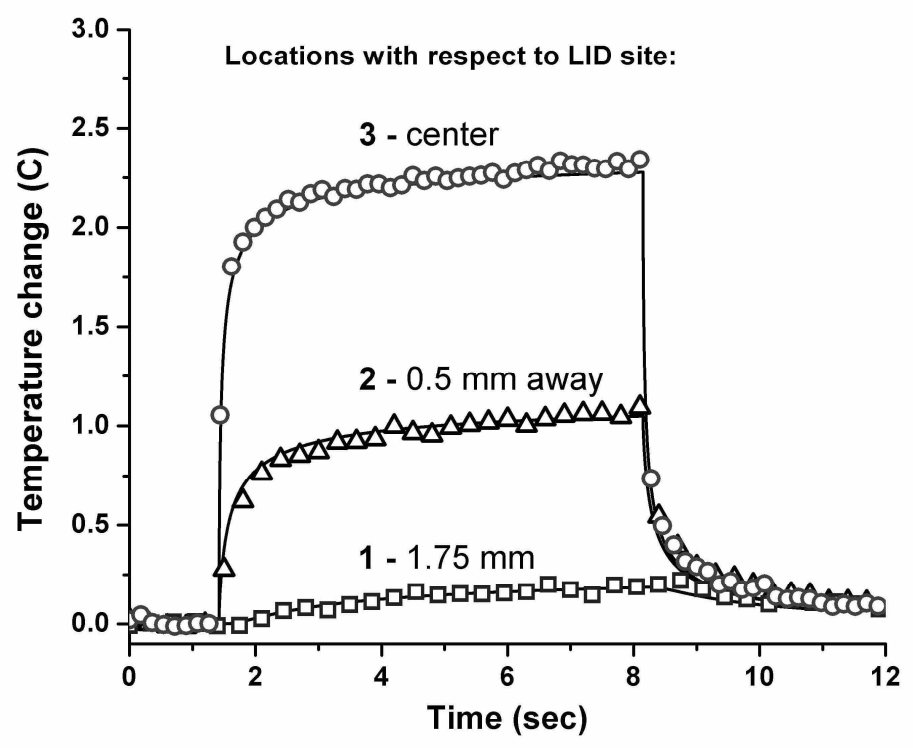

Figure 3. Changes in surface temperature at three locations was measured prior to, during and after a short exposure to $\mathrm{cw}$, UV laser (at $2.6 \mathrm{~W}$ ) of a typical LID site. Solid lines represent numerical fits based on a thermal diffusion model (see text).

We have constructed a thermal diffusion model to fit the dynamics of the local surface temperature upon irradiation and derive the fraction of the incident energy deposited within the LID site. Specifically, we considered exponential absorption of a Gaussian heat source profile with HW1/eM of $200 \mu \mathrm{m}$ (taking into account scatteringinduced broadening) in a $5 \mu \mathrm{m}$-thick layer ${ }^{1}$ within the LID site $(500 \mu \mathrm{m}$-diameter in this case). The best fits to the data (shown by the solid lines in Fig. 3) were obtained assuming a uniform absorption coefficient of $96 \pm 3$ $\mathrm{m}^{-1}$ of the laser modified material. The same experiment and numerical fitting of the data were repeated at five different LID sites and revealed values of the absorption coefficient of laser-modified material in the range $50-150 \mathrm{~m}^{-1}$. The spread in the values of the estimated absorption can be understood in terms of site-to-site variation in damage morphology as well as uncertainty in the depth of the absorption layer of modified material.

A second set of experiments was conducted to investigate the effects of prolonged UV laser illumination (15 mins) on the absorption properties of the modified material. During this time, a more significant heating of the entire sample was achieved. Simultaneously, the fluorescence microscope revealed a decrease in the fluorescence intensity originating at the damage site versus time. At the end of the extended exposure period, the laser was turned off and the relaxation of the sample's surface temperature back to room temperature was observed. In this irradiation regime, we have performed simultaneous dual imaging to monitor both thermal (not shown here) and fluorescence intensity of previously non-irradiated LID sites approximately once every minute up to one 
hour with $0.5 \mathrm{msec}$ integration time. During the relaxation period, the laser was briefly turned on to acquire fluorescence images.
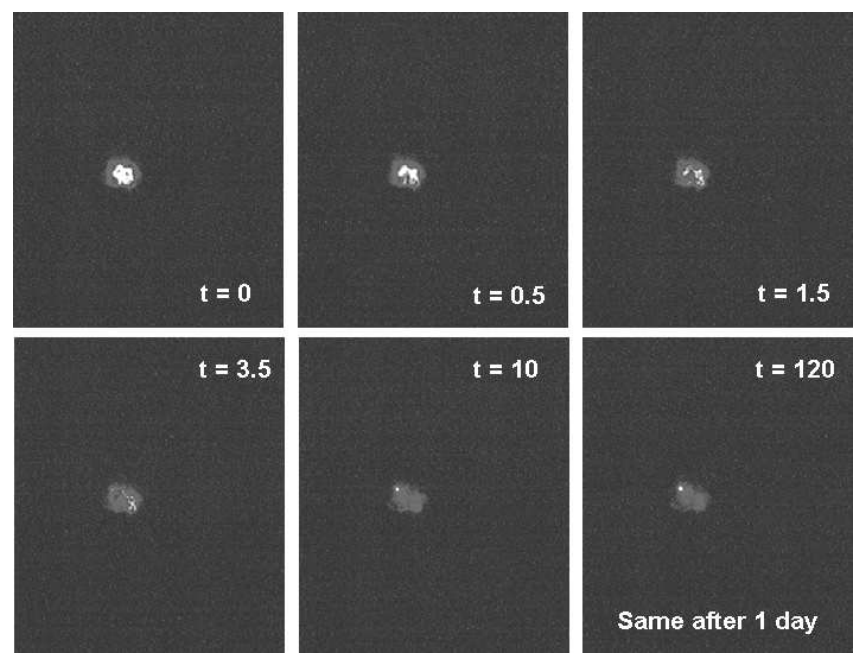

Figure 4. Fluorescence images of an LID site during and after extended exposure (15 mins) to constant UV laser exposure at $2.7 \mathrm{~W}$ versus time ( $\mathrm{t}$ in minutes).

Figure 4 illustrates the evolution of the fluorescence signal originating at a fresh LID site during and after extended exposure to UV laser at $2.7 \mathrm{~W}$. We note that, during irradiation, the integrated fluorescence intensity rapidly decreases to $\sim 20 \%$ of its value prior to irradiation. After the laser is turned OFF, within 15 mins, the surface temperature at the site relaxes back to room temperature but the fluorescence signal remains low, even after one day, suggesting that extended UV laser exposure induces irreversible photochemical reactions that in turn lead to significantly lower absorption within the modified material. This assumption was verified by performing short laser exposure experiments (few seconds) at previously non-irradiated damage sites to measure the transient surface temperature both prior to and after extended UV irradiation. The results of the latter tests are illustrated in Fig. 5. Indeed, we confirmed that the maximum temperature rise at LID sites following extended exposure and relaxation periods is also reduced to $\sim 20 \%$ of its value prior to irradiation, consistent
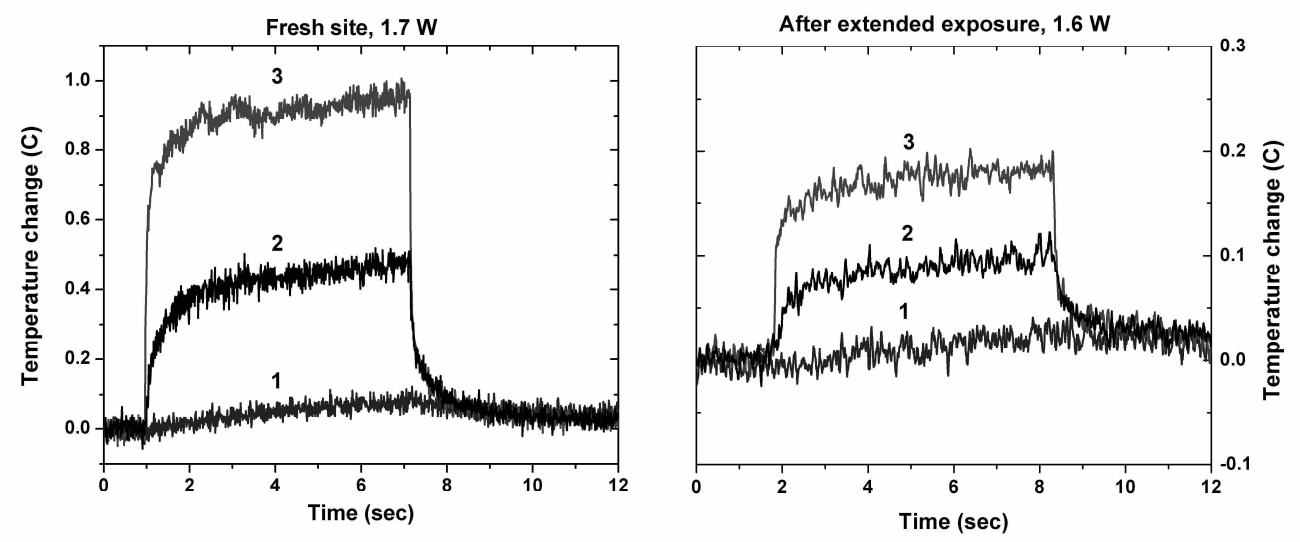

Figure 5. Comparison of transient surface temperature observed from short laser exposure experiments (at $1.7 \mathrm{~W}$ and $1.6 \mathrm{~W}$ ) on a previously non-irradiated LID site before and after extended UV exposure at $2.7 \mathrm{~W}$ and relaxation (left and right panels, respectively). Locations $\mathbf{1}, \mathbf{2}$, and $\mathbf{3}$ are similar to those indicated in Fig. 3. 
with a reduced absorption within the modified material. This effect may be related to previous studies where it has been suggested that thermal annealing of fused silica leads to passivation of the defects. ${ }^{2,3}$ Similarly, a reduction in the nonlinear absorption at damage sites has been reported following prolonged exposure to pulsed laser radiation. ${ }^{4}$ It is not clear whether or not the same absorbing defect species and/or absorption mechanisms are involved in these processes.

We can use the estimated linear absorption coefficient derived in the beginning of this section to predict the increase in temperature at the LID site under nanosecond, pulsed excitation assuming linear absorption at ICF laser fluences. This scenario is important as it relates to the amount of damage growth upon subsequent laser exposure. For example, if we consider the average absorption coefficient of the modified material of $100 \mathrm{~m}^{-1}$ and a $10-\mathrm{J} / \mathrm{cm}^{2}, 10-\mathrm{ns}$ incident laser pulse at $355 \mathrm{~nm}$ (Gaussian spatial beam profile with FHW1/eM of 500 $\mu \mathrm{m})$, the model predicts an upper bound on the induced temperature rise at the LID site of $\sim 3{ }^{\circ} \mathrm{C}$. Thus, the absorption cross-section derived above would prove insufficient to cause a significant increase in the temperature of the modified material under nanosecond, pulsed excitation (expect $\sim 1000{ }^{\circ} \mathrm{C}$ or higher ${ }^{5}$ ) and it suggests that absorption in the damaged material may be more localized and/or that nonlinear absorption mechanisms may be involved.

We have also performed thermal imaging of damage sites using ns, pulsed excitation at $355 \mathrm{~nm}$. For these experiments, we have synchronized the acquisition of thermal images with the ns laser pulse and set the integration window to $50 \mu \mathrm{s}$. Under these excitation conditions, we were able to detect changes in samples surface temperature only at laser fluences above the damage growth threshold $\left(\sim 10 \mathrm{~J} / \mathrm{cm}^{2}\right)$. At these high fluences, we observed shot-to-shot variation in temperature distribution across the LID site with consecutive pulses at $10 \mathrm{~Hz}$ repetition rate, indicative of non-uniform energy deposition within the modified material associated with changes in site morphology during growth. Moreover, we could not resolve the abrupt changes in temperature at LID site during the ns-pulse. Hence, the perceived temperatures were on the order of $\sim 100{ }^{\circ} \mathrm{C}$, much less than the expected temperatures to be reached during growth.

\section{SUMMARY}

We have obtained information on the energy deposition under UV, cw excitation of laser-modified fused silica at damage sites via linear absorption mechanisms. Based on a thermal diffusion model, we estimated 1) the linear absorption coefficient at 351-nm of the modified material, $\alpha=50-150 \mathrm{~m}^{-1}$, and 2) the temperature increase under ns, pulsed excitation via linear absorption mechanisms, $\delta \mathrm{T}=3{ }^{\circ} \mathrm{C}$. These low values suggest that either absorption is more localized or a nonlinear absorption mechanism governs the damage growth process. In addition, we have observed irreversible changes in the absorption at LID sites after extended UV laser exposure.

\section{ACKNOWLEDGMENTS}

This work was performed under the auspices of the U.S. Department of Energy by University of California, Lawrence Livermore National Laboratory under contract no. W-7405-Eng-48.

\section{REFERENCES}

1. M. A. Stevens-Kalceff and J. Wong, "Distribution of defects induced in fused silica by ultraviolet laser pulses before and after treatment with a $\mathrm{CO}_{2}$ laser," J. Appl. Phys. 97, p. 113519, 2005.

2. M. Watanabe, S. Juodkazis, H.-B. Sun, S. Matsuo, H. Misawa, M. Miwa, and R. Kaneko, "Transmission and photoluminescence images of three-dimensional memory in vitreous silica," Appl. Phys. Lett. 74, pp. 39573959, 1999.

3. S. Juodkazis, M. Watanabe, H.-B. Sun, S. Matsuo, J. Nishii, and H. Misawa, "Optically induced defects in vitreous silica," Appl. Surf. Sci. 154, pp. 696-700, 2000.

4. A. D. Walser, S. G. Demos, M. Etienne, and R. Dorsinville, "Nonlinear optical absorption in laser modified regions of fused silica substrates," Opt. Comm. 240, pp. 417-421, 2004.

5. C. W. Carr, H. B. Radousky, A. M. Rubenchik, M. D. Feit, and S. Demos, "Localized dynamics during laser-induced damage in optical materials," Phys. Rev. Lett. 92, p. 087401, 2004. 